\title{
PAROXYSMAL VENTRICULAR STANDSTILL
}

\author{
BY \\ HAROLD COOKSON
}

From the General Hospital, Poole

Received January 1, 1952

Among the various rhythms reported in the course of a Stokes-Adams attack, one of the rarest is standstill of the ventricle as an episode during normal rhythm. Such a condition may be called paroxysmal ventricular standstill, and is to be distinguished from the condition of alternating complete block and normal rhythm to which the term paroxysmal heart block may be applied. Three cases of paroxysmal ventricular standstill have been observed, in two of which there was paroxysmal heart block also, and ventricular standstill was also provoked by carotid sinus pressure.

Weiss and Baker (1933) described several cases of total cardiac standstill, auricular standstlil, or extreme sinus bradycardia, and one of complete block in auricular fibrillation as a result of carotid sinus pressure: all of these had cardiovascular disease. Starling (1921) reported ventricular standstill on swallowing but no cardiographic records were made of the attacks. Yater and Williams (1928-29) described a case of complete cardiac and ventricular standstill with slow irregular P waves. The case of Lewis (1920) showed ventricular standstill with auricular rhythm preserved; this patient later developed a complete block. A case recorded by Alexander and Bauerlein (1936) had variations in the degree of heart block with changes of posture but not with carotid sinus pressure. Holmes and Weill (1945) recorded mild grades of heart block in healthy men when they lay down; carotid sinus pressure caused a decrease in the P-R interval. Turner (1948) recorded the case of a man of 61 with cardiac asystole with pressure on either carotid sinus, whose attacks ceased after bilateral carotid sinus denervation. Parkinson et. al. (1941) recorded 5 examples of ventricular standstill, and collected 28 others that had been reported. Among all these there was only one in which the standstill was immediately followed by normal rhythm with unimpaired A-V conduction; this patient showed complete heart block on another day. Isolated cases of ventricular standstill are mentioned in two or three textbooks.

\section{CASE REPORTS}

Three patients have been observed with attacks of ventricular standstill, two of whom had normal rhythm and conduction before and after the standstill. In two of them the attacks could be provoked in various ways, in addition to those that had no apparent provocation.

Case 1. A woman of 82 had retinal hæmorrhage and diabetes for which she was having an injection of 50 units of insulin daily. For seven months she had been having syncopal and convulsive attacks. Physical examination was normal except for the retinal hæmorrhage. A cardiogram showed normal rhythm, right bundle branch block, but no delay in A-V conduction. As the record was being completed with lead IV $R$, the ventricle suddenly stopped with immediate loss of consciousness and a generalized convulsion after 5 seconds (Fig. 1); the movement of the record off the paper indicates the beginning of limb movements. The recording, when resumed at the end of the convulsion shows normal rhythm and conduction exactly as before except that the rate was slightly quicker. As weeks might elapse between this patient's attacks, it was most fortunate that one occurred during the only tracing taken. No further information about the subsequent history was available except that she died a few months later. 


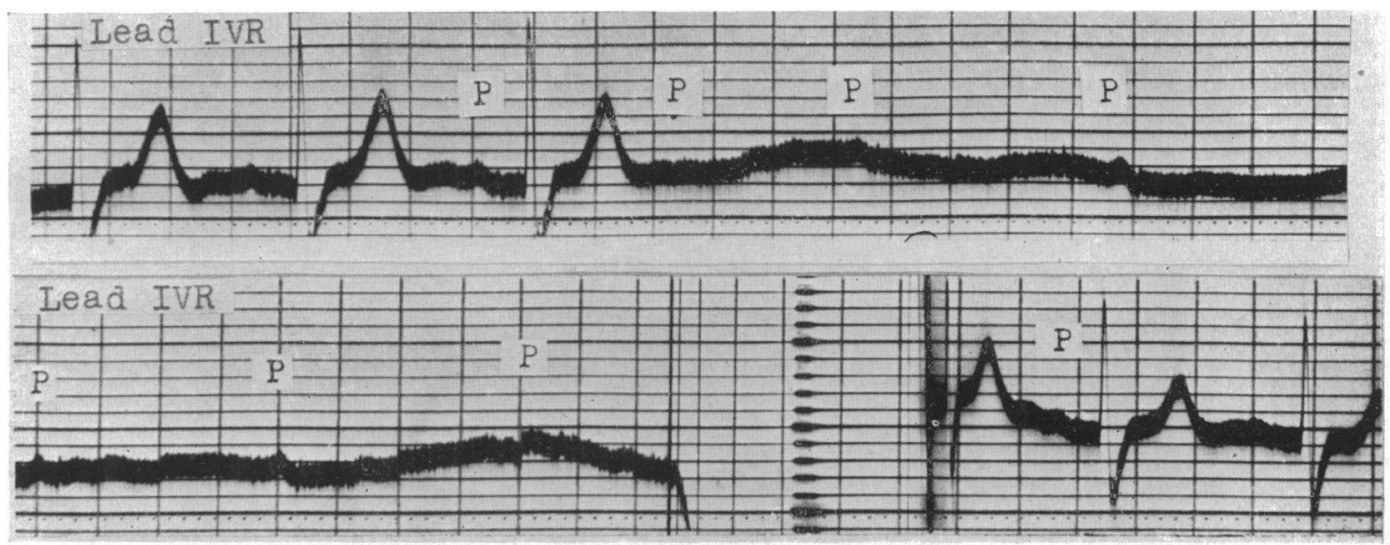

FIG. 1.-Case 1. Woman, aged 82. Spontaneous ventricular standstill; generalized convulsion 5 seconds after the ventricle stops; at end of attack ventricular beating resumed with normal P-R interval.

Case 2. A man, aged 78, with a history of rheumatic fever in childhood and of an illness diagnosed as endocarditis when aged 51 , gave a history of " black-outs" for ten years, but only at very long intervals for the first nine years. He developed a gastric ulcer at the age of 77 at which time his syncopal attacks became more frequent. At the apex there was an extra heart sound due to the latent block, but otherwise there was no sign of cardiovascular disease. Normal rhythm with prolonged A-V conduction was usually present (Fig. 2) but sometimes partial or complete

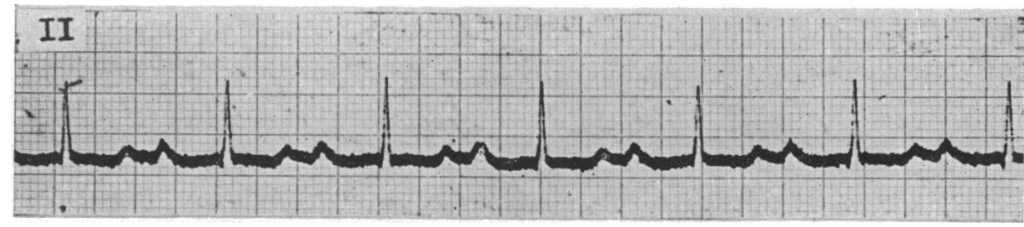

Fig. 2.-Case 2. Man, aged 78. Normal rhythm; P-R interval about $0 \cdot 3$ sec.

heart block. An attack of unconsciousness with convulsion was witnessed in which the peripheral pulse disappeared. Normal rhythm was recorded before and after it. Then very light pressure on the right carotid sinus produced an increase in A-V conduction time for one cycle (Fig. 3) and then ventricular standstill; the auricular rate gradually increased from 65 to 90 a minute, and after about 8 seconds, convulsive movements of the limbs were large enough to jerk the recording pen off the paper. After a few seconds, ventricular activity returned with a rate of about 90 , the A-V conduction time being about $0.3 \mathrm{sec}$, the same as before the attack. Ventricular contractions returned some time before consciousness was fully regained and before limb movements ceased. On other occasions heavy and prolonged carotid sinus pressure would cause only one or two dropped beats with very slight auricular slowing (Fig. 4). This figure also shows a record taken while the patient turned his head vigorously from side to side; it coincided with an auricular extrasystole which was blocked. On yet another occasion when left carotid sinus pressure was without effect, pressure on the right one caused an extreme sinus bradycardia with ventricular escape (Fig. 5). After a ventricular extrasystole, sinus beating was resumed at almost the usual rate in spite of carotid sinus pressure being maintained. When complete block was present, carotid sinus stimulation seemed to be without any effect. Fig. 6 shows complete block with an occasional conducted beat; this last occurs when $P$ falls about $0 \cdot 3 \mathrm{sec}$. before QRS. Then pressure on both carotid sinuses successively was exerted; the middle tracing shows the independent 


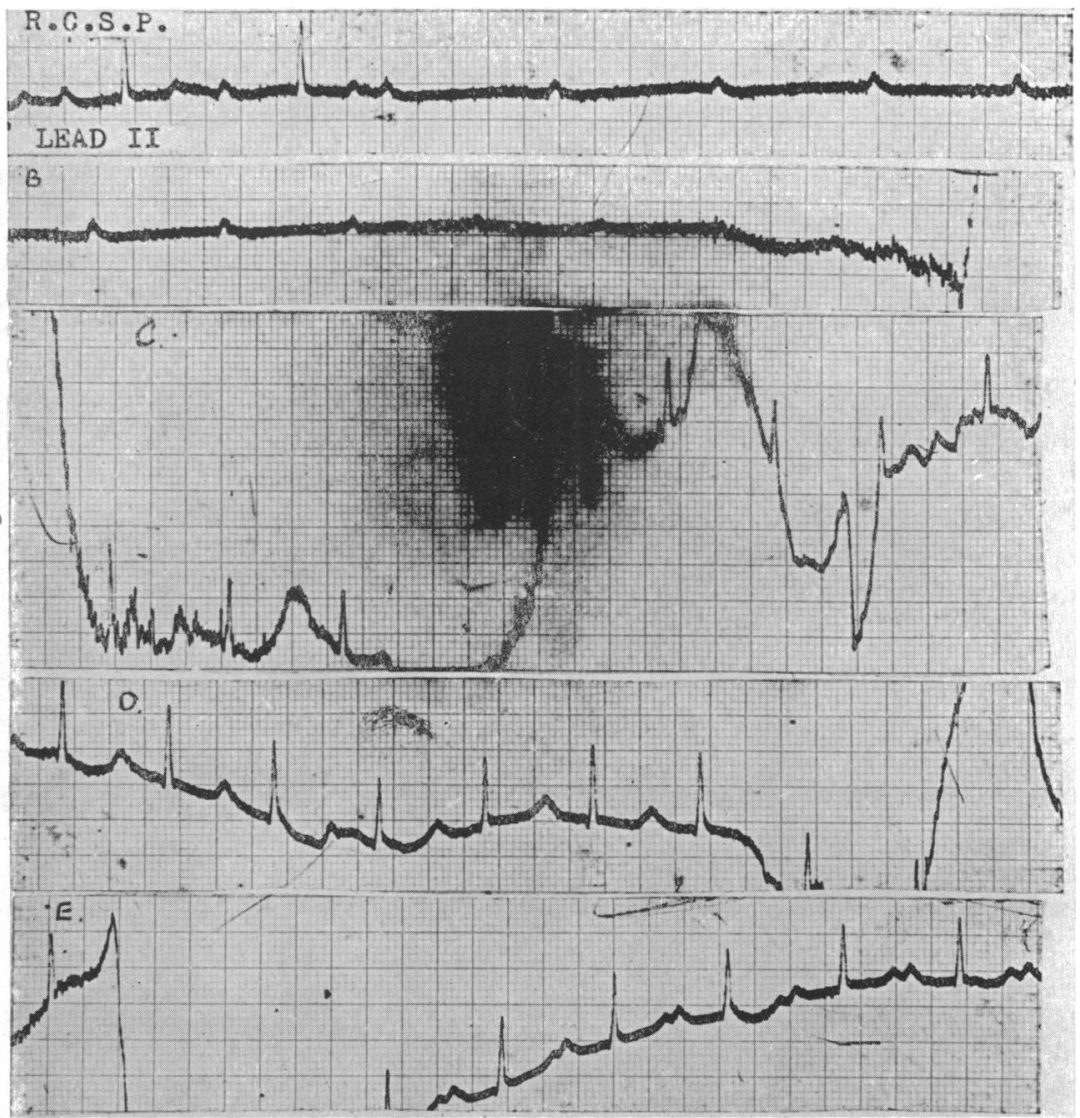

Fig. 3.-Case 2. Light pressure on the right carotid sinus causes increase in the P-R interval for one cycle and then ventricular standstill, leading to convulsion after 8 seconds; a few seconds later $A-V$ rhythm returns with the P-R interval the same as before the attack.

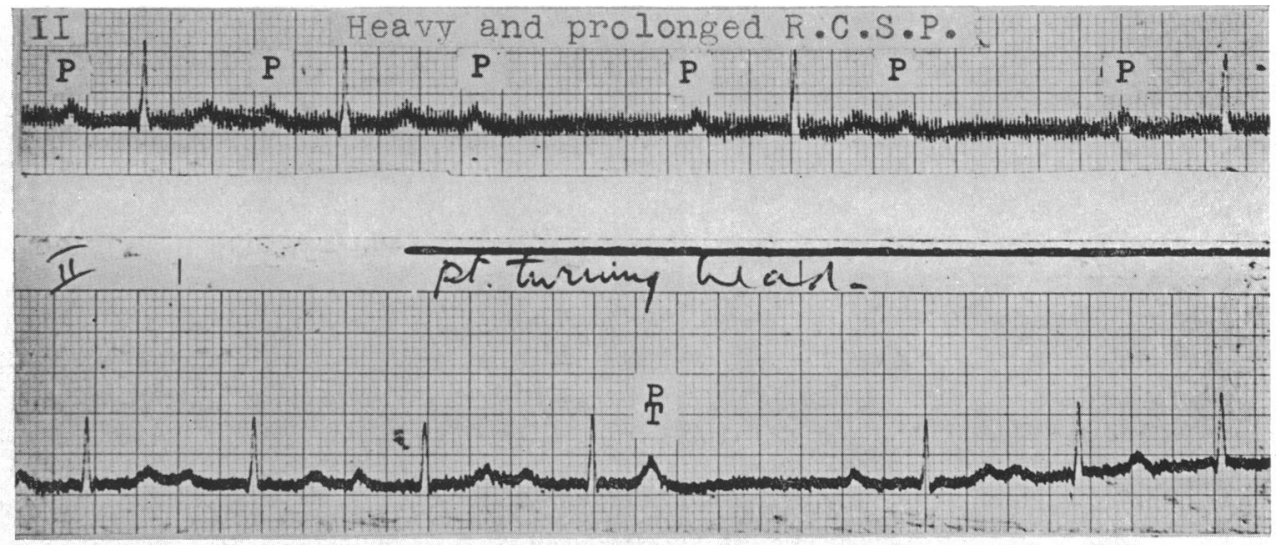

Fig. 4.-Case 2. Upper tracing: heavy and prolonged right carotid sinus pressure caused only one or two dropped beats. Lower tracing: vigorous head-turning by the patient coincided with a blocked auricular extrasystole. 


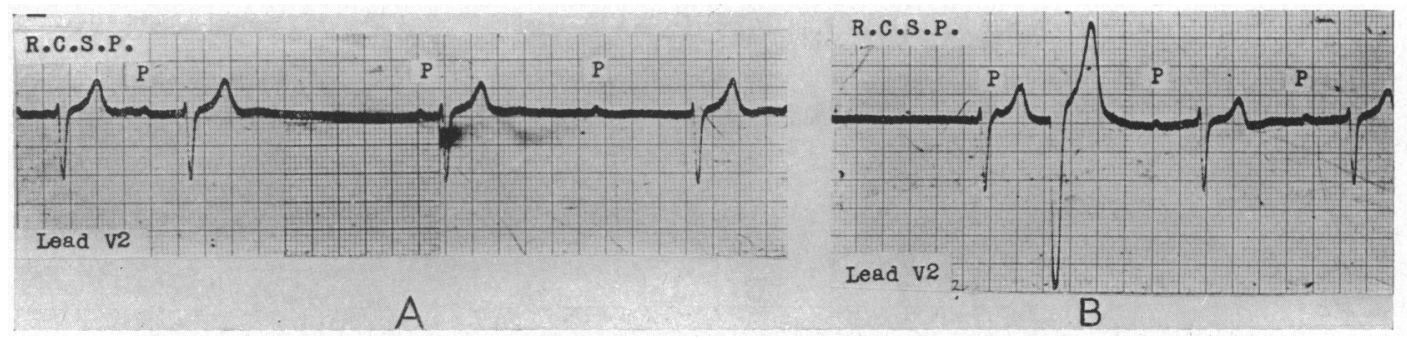

FIG. 5.-Case 2. (A) Right carotid sinus pressure caused sinus bradycardia and ventricular escape. (B) After a ventricular extrasystole, normal rhythm and rate return in spite of continued right carotid sinus pressure.
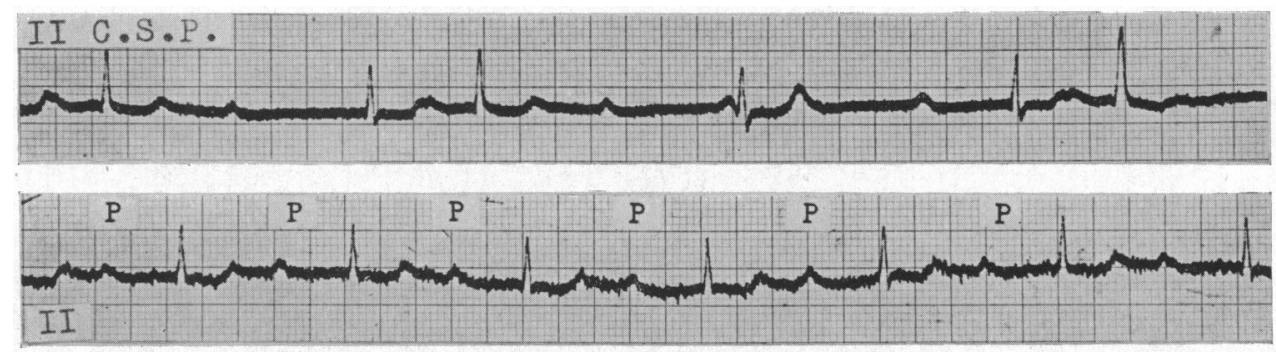

Fig. 6.-Case 2. Upper tracing: complete heart block with occasional A-V conduction; carotid sinus pressure either left or right has no effect. Lower tracing: return of normal rhythm, P-R interval 0.4 sec., soon after carotid sinus pressure had been stopped.

auricular and ventricular rhythms maintained as before. After all this pressure on the neck had stopped, the patient was in a distressed condition, but normal rhythm had returned with a P-R interval of $0.4 \mathrm{sec}$. (lower tracing, Fig. 6). Treatment of this patient with eumydrine, ephedrine, and digitalis in turn had no certain effect on the frequency of the Stokes-Adams attacks.

Case 3. A man, aged 69, subject to attacks of loss of consciousness since 1938, was said to have had rheumatic fever when 7 years old; there was no evidence of rheumatic heart disease, but some hypertension with left ventricular hypertrophy was present. When first seen in 1945, he had normal rhythm and it was not until 1948 that a record showing complete block was obtained. About this time, during the taking of a routine tracing which showed normal rhythm, a ventricular extrasystole occurred and this was immediately followed by ventricular standstill, loss of consciousness, and a convulsion (Fig. 7). Immediately after the attack, the tracing was resumed and showed normal sinus rhythm and the A-V conduction within normal limits. The patient himself preferred his pulse slow (complete block) rather than quick (normal rhythm) as then he found he had fewer attacks and he had noticed that if he raised his left arm, he would lose con-

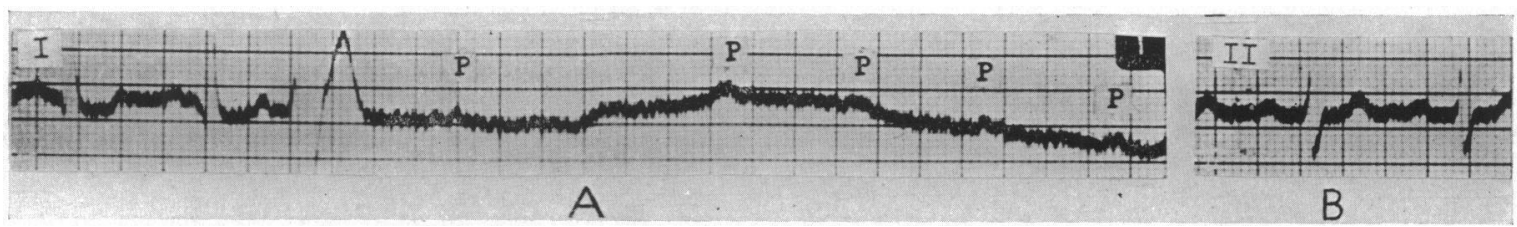

Fig. 7.-Case 3. Man, aged 69. Normal rhythm, normal P-R interval. (A) Ventricular standstill follows a ventricular extrasystole (lead I), causing a Stokes-Adams attack. (B) Normal rhythm and conduction are resumed immediately after the attack. 


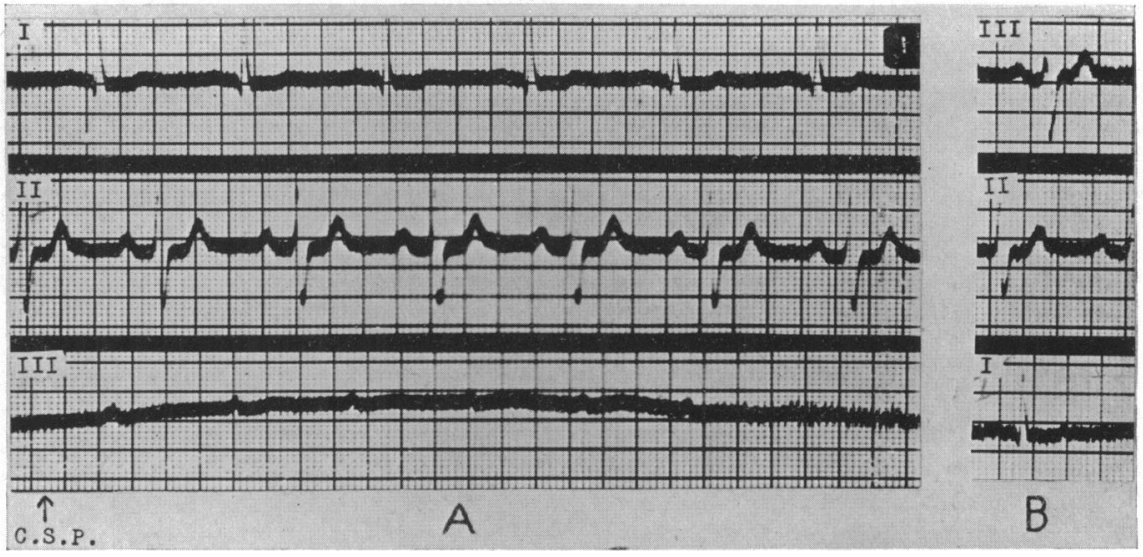

FIG. 8.-Case 3. (A) Ventricular standstill produced immediately by carotid sinus pressure. (B) Normal rhythm and conduction recorded as soon as the attack was over.

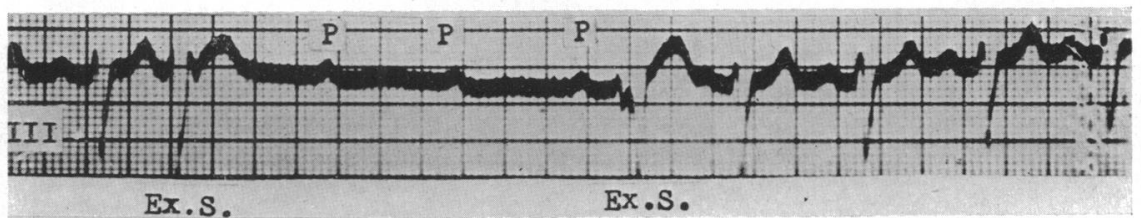

Fig. 9.-Case 3. Ventricular standstill for three cycles following an extrasystole; the first beat after the standstill is also aberrant.

sciousness but not if he raised his right arm. Carotid sinus pressure was tried first on the right without effect, and then on the left with immediate ventricular standstill (Fig. 8). The almost instantaneous effect of the pressure is shown in the record, pressure on the carotid sinus coinciding with depression of the lever to start the recording drum. The beginning of limb movements is shown by the unsteadiness of the tracing after about 5 seconds. During this time the auricular rate increased from 75 to 100 a minute. The tracing was resumed as soon as possible after the attack and was exactly the same as before it, with sinus rhythm, rate about 75 and normal A-V conduction time. For some months all records showed normal rhythm; then one was obtained showing ventricular standstill for three cycles after a ventricular premature beat, the resumption of normal rhythm being preceded by another ventricular premature contraction (Fig. 9).
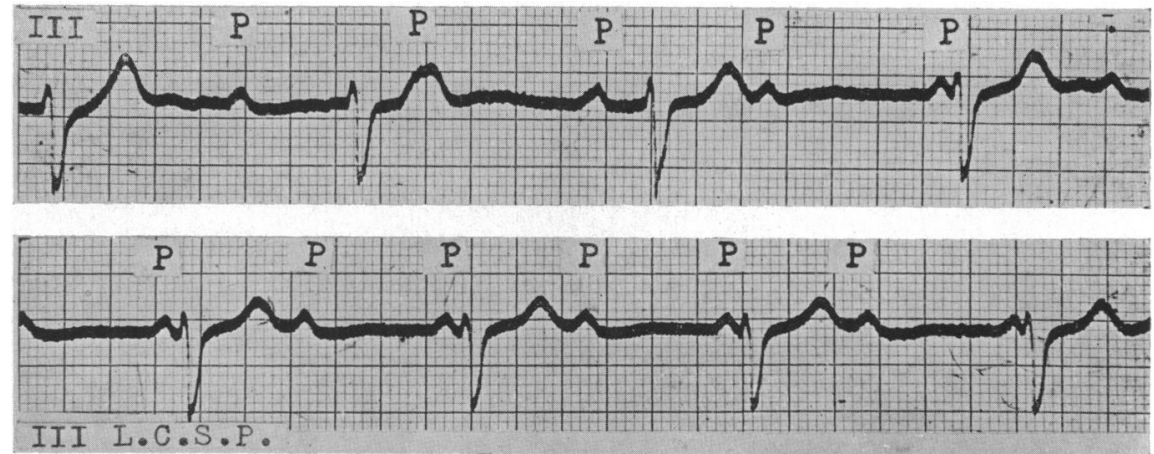

Frg. 10.-Case 3. Upper tracing: complete heart block. Lower tracing: left carotid sinus pressure causes no auricular or ventricular slowing. 

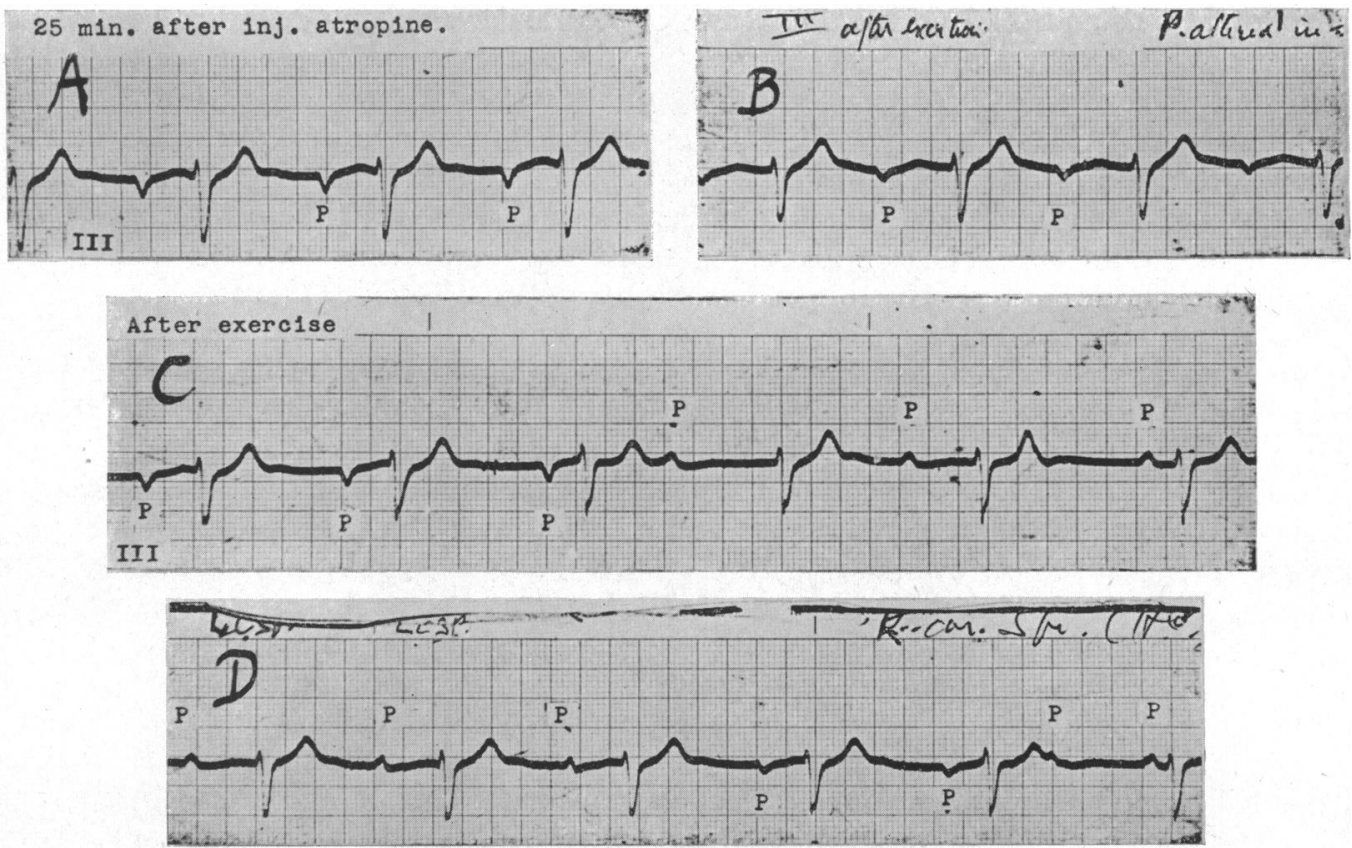

Fig. 11.-Case 3. (A) After injection of atropine gr. 1/100, complete heart block with auricular and ventricular rates almost the same; $P$ inverted. (B) After exertion $P$ altered in shape. (C) After more exertion $\mathbf{P}$ at first negative becomes upright; 2:1 sino-auricular block; complete A-V block. (D) Complete block and $2: 1$ sino-auricular block; carotid sinus pressure causes temporary inversion of $\mathrm{P}$; sino-auricular block disappears at end of tracing.

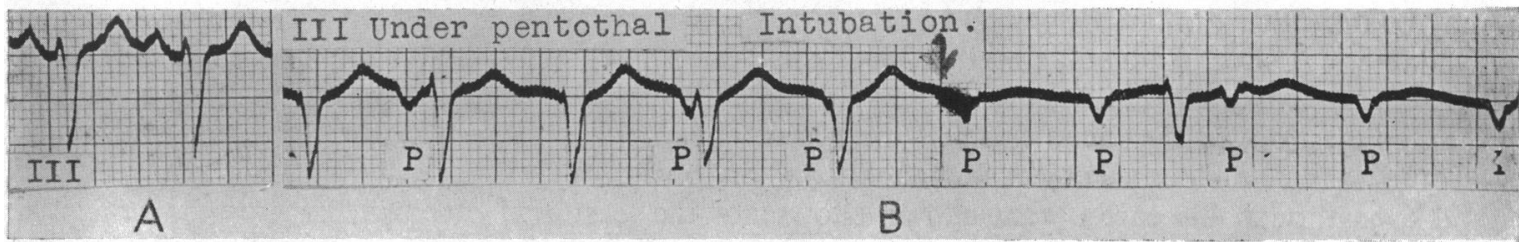

Fig. 12. Case 3. (A) Normal rhythm and normal conduction, rate 105 a minute. (B) Under pentothal anæsthesia, ventricular rate unchanged, $P$ negative; probably nodal rhythm: passing of tracheal tube (shown by arrow) produced complete block.

The following day, auricular fibrillation was recorded at which time neither arm raising nor carotid sinus pressure had any effect. When complete block was present, carotid sinus pressure whether left or right was incapable of causing ventricular standstill, nor did it slow the auricle (Fig. 10). Two to one sino-auricular block occurred from time to time; it would appear after exertion and after an injection of atropine (Fig. 11); at other times the $\mathrm{P}$ wave became inverted or biphasic. While waiting to be given an anæsthetic, this patient had sinus tachycardia with normal A-V conduction (Fig. 12, A). After pentothal P became inverted, the ventricular rate being much the same. At the moment a McGill's tube was passed through the nose into the trachea, there was a transition to complete A-V dissociation (Fig. 12, B).

\section{Discussion}

The commonest causes of the Stokes-Adams attack are believed to be: (1) transition from normal rhythm to high-grade block; (2) slowing of the idio-ventricular rhythm in the course of complete block; and (3) abnormal ventricular rhythms such as ventricular tachycardia and fibril- 
lation followed by standstill. Of the three cases of ventricular standstill described here occurring as an episode in the course of normal rhythm, two showed varying degrees of heart block from time to time. They had also extreme sensitivity of the carotid sinus at times, but this was only found when normal rhythm was present. Then quite slight pressure on the carotid sinus-one side was more responsive than the other-was capable of arresting the ventricle within about a second, followed immediately by loss of consciousness and then within 5 to 8 seconds by tonic contraction of the limbs. The term loss of consciousness is not a precise one, but it is certain that immediately after the ventricle stopped beating, the patients began to lose consciousness, while the onset of tonic limb contractions can be measured accurately from the movement of the baseline of the tracing. The immediate impairment of consciousness may not be entirely due to the fall in cerebral blood flow, and it is possible that a direct stimulation of the cerebral circulation plays a part as suggested by Weiss and Baker (1933) to explain cases in which carotid sinus stimulation caused syncope without slowing of the heart rate or fall in blood pressure. The extreme sensitivity of the conducting tissues to nerve impulses is shown by the induction of mild attacks by merely raising the arm, or changing posture. These events occurred only when normal rhythm was present and even then the response to carotid sinus pressure varied much from day to day and even from hour to hour. However, the position was very different when complete block was present; then carotid sinus stimulation was without effect on the ventricle and usually on the auricle also, though it might slow its rate a little or displace the site of impulse formation. It appears that when normal rhythm is present, the reflex from the carotid sinus acts on the upper part of the bundle of His and is temporarily incapacitated, although immediately before and after it conducts perfectly. On the other hand, when complete block is present, the centre governing the ventricle, situated lower in the bundle, is immune from the effects of the reflex. An interesting observation is the onset of ventricular standstill after a ventricular extrasystole. Might this result from fatigue of the bundle by the premature retrograde stimulation, or alternatively might a nervous impulse simultaneously act upon the ventricle and the bundle to produce both effects?

The diagnosis of epilepsy from loss of consciousness due to paroxysmal ventricular standstill may be difficult from the history alone, even if a description of the attacks by a good eye-witness is available. A short standstill simulates petit mal very closely, but a longer one leading to complete loss of consciousness and convulsion will usually differ from grand mal in the facial pallor rather than congestion, in the facial flush as the attack ends, and in the more rapid return of full consciousness. The elderly subject with a history of loss of consciousness, even over many years, and even though his cardiogram at first shows not the least sign of block, may yet be suffering from paroxysmal ventricular standstill or other kind of heart block. For such a patient, carotid sinus pressure might settle the diagnosis, though this is hardly a method to be used indiscriminately. Apart from this, the true diagnosis will probably be revealed by continued observation, as sooner or later ordinary forms of auriculo-ventricular block will make their appearance.

It used to be thought that the heart block of chronic heart disease was usually permanent, but it is now clear that in many the rhythm passes back and forth from block to normal over long periods of years, the duration of each varying from seconds to months. In addition to the basic structural lesion, therefore, other factors must be involved and among these are reflexes from the carotid sinus, pharynx, and esophagus, and perhaps impulses arising in the brain itself as a result of emotional disturbance.

\section{REFERENCES}

Alexander, H. L., and Bauerlein, T. C. (1936). Amer. Heart J., 11, 223.

Holmes, J. H., and Weill, D. R. (1945). Amer. Heart J., 30, 291.

Lewis, T. (1920). The Mechanism and Graphic Registration of the Heart Beat. London, p. 358.

Parkinson, J., Papp, C., and Evans, W. (1941). Brit. Heart J., 3, 171.

Starling, H. J. (1921). Heart, 8, 31 .

Turner, R. W. D. (1948). Quart. J. Med., 17, 327.

Weiss, S., and Baker, J. P. (1933). Medicine, 12, 297.

Yater, W. M., and Williams, F. A. (1928-29). Amer. Heart J., 4, 280. 